\title{
SNPs, siRNA and DNAs
}

\section{Tools for gene and protein handling.}

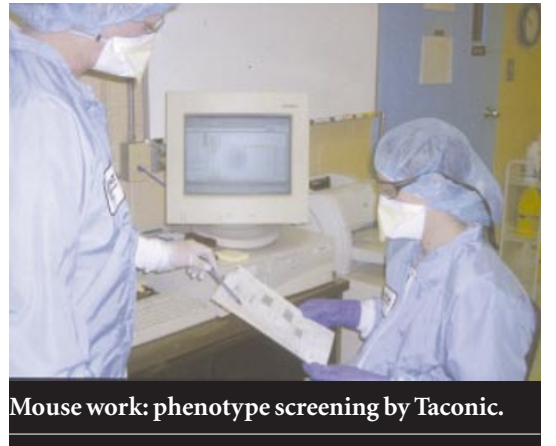

Phenotyping panels

Taconic Biotechnology www.taconic.com See your mice in a new light

A series of phenotyping panels from Taconic Biotechnology can be used to help determine the phenotype of gene expression in traditional and genetically altered laboratory mice. The General Screening Panel consists of necropsy, urinalysis, haematology and serum biochemistry tests to provide a view of the fundamental phenotype of the animal. A 'Histology' option adds tissue preservation and pathologist evaluation of the organs surveyed in the necropsy, while the 'Behaviour' option focuses on motor coordination and basic neurologic behaviour of the animal. Finally, the 'Reproductive' option appraises the breeding performance of the strain.

\section{Recombinant Proteinase K}

\section{Roche Applied Science}

www.roche-applied-science.com

PCR grade now available

Roche's Recombinant PCR-Grade Proteinase $\mathrm{K}$ is tested as nuclease-free, function-tested and virtually free of DNA ( $<10$ pg per mg) and other contaminants found in native preparations. It is active over wide $\mathrm{pH}$ $(4.0-10.0)$ and temperature $\left(25-50{ }^{\circ} \mathrm{C}\right)$ ranges. This Proteinase $\mathrm{K}$ preparation is a high-activity endopeptidase that completely inactivates nucleases during manual or automated purification of DNA or RNA for use in PCR, RT-PCR, cloning, sequencing and many other applications.

\section{Montage PCR m96}

Millipore www.millipore.com

Small-volume PCR purification

Millipore's 96-well Montage PCR $\mu 96$ cleanup plates incorporate the company's sizeexclusion technology to process PCR reaction volumes from 1 to $150 \mu \mathrm{l}$. The microwell plate design is optimized to purify, concentrate and recover PCR products in as little as
$20 \mu l$. The 15 -minute vacuum-driven protocol removes primers $(>99 \%$ primer removal) and dNTPs in one step. The plates yield purified PCR product suitable for sensitive genotyping applications, direct sequencing and microarray spotting. In genotyping assays, the Montage plates are particularly useful for MALDI-TOF and CEbased detection methods because of the small reaction volumes. For microarray spotting applications, the plates can concentrate PCR products up to 7.5-fold with high recoveries. Montage plates are compatible with liquid-handling equipment.

\section{ProteomeLab PF 2D}

Beckman Coulter www.beckmancoulter.com Fractionation in two dimensions

The ProteomeLab PF 2D is an automated, two-dimensional fractionation system for high-resolution analysis of complex protein mixtures. In the first dimension, chromatofocusing separates the proteins according to their $\mathrm{pI}$, providing information that can be related to traditional isoelectric focusing techniques. Fractions from the first dimension are collected and automatically injected into a second dimension, which separates on the basis of hydrophobicity. This 'in-solution' approach delivers liquid fractions for further study. The software displays both one- and two-dimensional maps of the expressed protein profiles in easy-to-read formats.

\section{SNP Assistant}

\section{Asper Biotech/ BioData}

www.asperbio.com/ www.biodata.ee

The generation game

Genotyping specialists Asper Biotech and bioinformatics company BioData are collaborating on a 'next generation' tool for han-

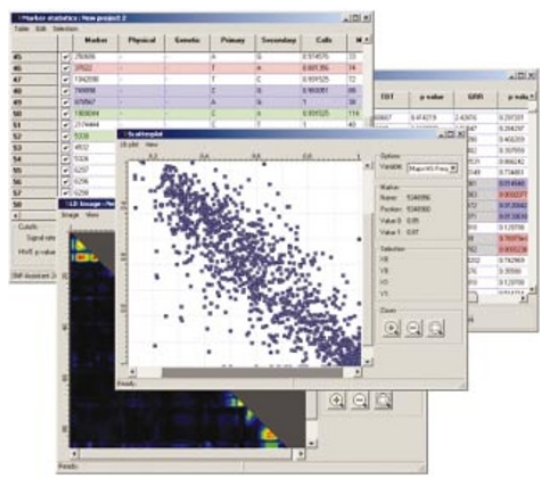

It's a SNP: download a demo from BioData. 
dling SNP genotyping data. SNP Assistant is an easy-to-use tool for single nucleotide polymorphism (SNP) management, validation and analysis. Functions include marker and sample validation, case-control and TDT tests, LD calculation and publication level visualization, relationships testing and comparison of datasets. SNP Assistant is not platform-dependent and handles genotyping results from all major technology platforms, enabling groups with different technologies to work in similar environments. A fully functional trial version (time restricted) of the software is available from BioData's website www.biodata.ee.

\section{Platinum PCR Supermix 96}

\section{Invitrogen} www.invitrogen.com Try another Taq

Invitrogen's Platinum SuperMix 96 for highthroughput PCR is a ready-to-use, scalable mixture of Taq DNA polymerase, anti-Taq antibodies, salts, magnesium and dNTPs. The inclusion of monoclonal antibodies that inactivate Taq DNA at low temperatures increases specificity. The mixture is prealiquoted into 96-well skirted or non-skirted perforated plates.

\section{Amplifluor}

Flowgen www.flowgen.co.uk SNP genotyping with a signal advantage

Flowgen's Amplifluor system for genotyping single nucleotide polymorphisms (SNPs) uses a dual-signal process with two fluorescently labelled primers to distinguish between heterozygous and homozygous alleles. Discrimination of alleles in a single closed tube is achieved with a pair of allelespecific primers with different tail sequences. Depending on the genotype of the sample, a mixed signal of fluorescein and sulphorhodamine fluorescence is observed for a heterozygote, while a single signal from either is observed for a homozygote. Two Amplifluor kits are available — one for assay develop- ment, which is aimed at new users and has a capacity of 100 reactions, and one for highthroughput screening that can carry out 500 reactions and is designed for investigators who are familiar with the Amplifluor UniPrimer system and SNP genotyping technology. Only one reaction per SNP is needed and the system can use reactions containing as little as $400 \mathrm{pg}$ of genomic DNA.

\section{Dicer siRNA generation kit}

\section{Gene Therapy Systems}

www.genetherapysystems.com

A little interference

The Dicer siRNA generation kit can be used to generate a large number of small interfering RNAs (siRNAs) from full-length target genes. It eliminates the trial and error associated with synthetic siRNA and siRNA expression vectors by using recombinant human DICER enzyme to cleave in vitro transcribed dsRNA templates into a pool of 22 bp siRNAs. The kit contains everything required for preparing double-stranded RNA, RNA cleavage, siRNA purification and transfection.

\section{Perfectprep}

Eppendorf www.eppendorf.com BACPAC kit

The Perfectprep BAC 96 purification kit from Eppendorf isolates 96 individual BAC, PAC, fosmid or cosmid clones in parallel. Its trapping technology generates high-quality DNA with minimal shearing or chromosomal contamination, optimizing the accuracy of spectrophotometer readings and improving downstream applications such as sequencing (average read length $>600$ bases), fingerprinting, mutagenesis and PCR. Beginning with the pelleted bacteria, 96 samples can be processed in 60 minutes, or 192 samples in 75 minutes. There is no need for precipitation or resuspension of the purified DNA, so the eluate can be used immediately.






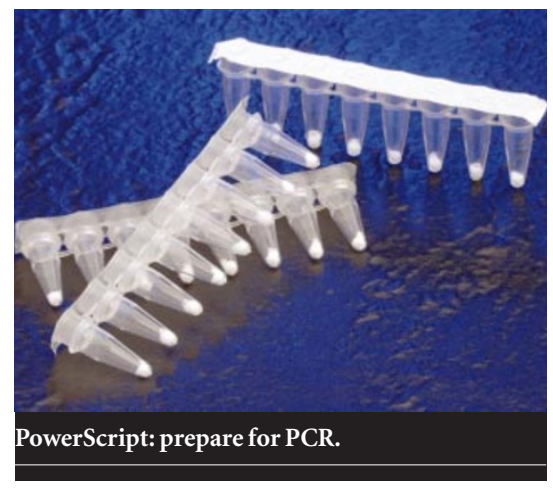

D Sprint PowerScript RT

BD Biosciences Clontech www.clontech.com Select reverse

This new product combines the BD PowerScript RT reverse transcriptase with a multiwell lyophilized delivery system. BD Sprint PowerScript Single Shots and BD Sprint PowerScript 96 Plate each contain a complete lyophilized master mix of BD PowerScript Reverse Transcriptase, dNTPs and buffer. To use, simply dissolve the lyophilized mixture using PCR-grade water containing diluted primer(s) and RNA and incubate. The makers say these kits provide consistent, RNase-free enzyme preparations ideal for cDNA synthesis and library construction, probe labelling, and all other RT-PCR applications.

\section{Soilmaster}

Epicentre www.epicentre.com Digfor information

The SoilMaster kit can be used to extract PCR-ready DNA from a variety of environmental samples. It uses a hot detergent lysis process combined with a chromatography step that removes organic inhibitors such as humic and fulvic acids that are known to coextract with DNA from soil and sediment samples. The extracted DNA can be used with the FailSafe PCR system to amplify bacterial, plant or fungal templates.

Utility cart

Labconco www.labconco.com

Mobilework station

Labconco's utility cart has two open shelves for small electrical equipment and a pair of drawers to hold small labware and supplies. The cart can be used for transporting lab equipment or as a compact work station. It is fitted with a power strip providing 115 volts, $60 \mathrm{~Hz}, 12$ amps, with six electrical outlets, a cord bracket and a three-wire cord with plug. The cart supports equipment weighing up to 400 pounds and has lockable casters.

\section{Beacon Designer}

\section{Premier Biosoft International}

www.premierbiosoft.com

Software for real time PCR

A new version of Beacon Designer has just been released. The program designs primers, TaqMan probes and molecular beacons optimized for quantitative PCR product detection and allele discrimination in multiplex experiments. Beacon Designer uses a sophisticated algorithm to analyse the results of BLAST search and template folding. The results of analysis are used to design highly

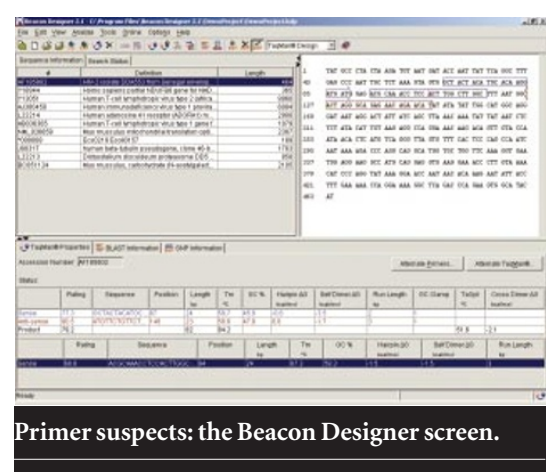

efficient and specific primers. With an intuitive interface and graphical display, the program makes real time assay design and analysis as easy as clicking a button.

\section{KDE GeneSense}

\section{Affibody/InforSense}

www.inforsense.com

Functional genomics

Affibody $\mathrm{AB}$ and InforSense have jointly introduced KDE GeneSense, a new system for interpreting experimental genomics, transcriptomics and proteomics data. GeneSense couples InforSense's Kensington Discovery Edition (KDE) discovery informatics environment with Affibody's data management, biological annotation and visual representation technology. GeneSense provides researchers with integrated and curated access to relevant information databases; links to KDE's flexible workflow system and powerful analytical tools; interpretation using different knowledge domains/vocabularies (or ontologies); and easy-to use Virtual Chip and Tree Map visualizers to facilitate the manipulation and display of ontologies.

\section{ProTeam}

Tecan

www.tecan.com Single-platform protein processing

ProTeam Advanced Digest is a scalable, flexible one-unit system for automated in-gel or in-solution protein digestion, peptide extraction and MALDI plate-spotting. The system can deliver samples to a range of target plates and can be integrated into Tecan's ProTeam product suite. It is based on Genesis liquidhandling and incorporates the company's DirectSpot technology with TecPrep 96 solidphase extraction plates to extend sensitivity. Sample volumes as low as $50 \mathrm{nl}$ can be used.

These notes are compiled in the Nature office from information provided by the manufacturers. 